\title{
UNITARY EQUIVALENCE TO A COMPLEX SYMMETRIC MATRIX: GEOMETRIC CRITERIA
}

\author{
LEVON BALAYAN AND Stephan RAMON GARCiA
}

Abstract. We develop several methods, based on the geometric relationship between the eigenspaces of a matrix and its adjoint, for determining whether a square matrix having distinct eigenvalues is unitarily equivalent to a complex symmetric matrix. Equivalently, we characterize those matrices having distinct eigenvalues which lie in the unitary orbit of the complex symmetric matrices.

Mathematics subject classification (2000): 15A57, 47A30.

Keywords and phrases: Complex symmetric matrix, complex symmetric operator, unitary equivalence, unitary orbit, UECSM.

\section{REFERENCES}

[1] N. Chevrot, E. Fricain, D. Timotin, The characteristic function of a complex symmetric contraction, Proc. Amer. Math. Soc., 135, 9 (2007), 2877-2886. MR2317964 (2008c:47025)

[2] J. A. Cima, W. T. Ross, W. R. Wogen, Truncated Toeplitz operators on finite dimensional spaces, Operators and Matrices, 2, 3 (2008), 357-369.

[3] S. R. Garcia, Conjugation and Clark operators, Contemp. Math., 393 (2006) , 67-111.

[4] S. R. Garcia, M. Putinar, Complex symmetric operators and applications, Trans. Amer. Math. Soc. 358 (2006), 1285-1315.

[5] S. R. Garcia, M. PUtinar, Complex symmetric operators and applications II, Trans. Amer. Math. Soc., 359, 8 (2007), 3913-3931.

[6] S. R. Garcia, W. R. Wogen, Some new classes of complex symmetric operators, (to appear: Trans. Amer. Math. Soc.). arXiv:0907.3761v1

[7] S. R. Garcia, W. R. Wogen, Complex symmetric partial isometries, J. Funct. Analysis, 257 (2009), $1251-1260$.

[8] T. M. Gilbreath, W. R. Wogen, Remarks on the structure of complex symmetric operators, Integral Equations Operator Theory, 59, 4 (2007), 585-590.

[9] R. A. Horn, C. R. Johnson, Matrix Analysis, Cambridge Univ. Press, Cambridge, 1985.

[10] D. SARASON, Algebraic properties of truncated Toeplitz operators, Oper. Matrices, 1, 4 (2007), 491526.

[11] J. TENER, Unitary equivalence to a complex symmetric matrix: an algorithm, J. Math. Anal. Appl., 341, 1 (2008), 640-648. MR2394112 